\title{
Backward compatibility effects in younger and older adults
}

\author{
Alan A. Hartley ${ }^{1}$ - François Maquestiaux ${ }^{2}$ - Sara B. Festini ${ }^{1,3} \cdot$ Kathryn Frazier $^{1,4}$ • \\ Patricia J. Krimmer ${ }^{1}$
}

Published online: 4 May 2016

(C) The Psychonomic Society, Inc. 2016

\begin{abstract}
In many dual-task situations, responses to the second of two tasks are slowed when the time between tasks is short. The response-selection bottleneck model of dual-task performance accounts for this phenomenon by assuming that central processing of the second task is blocked by a bottleneck until central processing of Task 1 is complete. This assumption could be called into question if it could be demonstrated that the response to Task 2 affected the central processing of Task 1, a backward response compatibility effect. Such effects are well-established in younger adults. Backward compatibility effects in older (as well as younger) adults were explored in two experiments. The first experiment found clear backward response compatibility effects for younger adults but no evidence of them for older adults. The second experiment explored backward stimulus compatibility and found similar effects in both younger and older adults. Evidence possibly consistent with some pre-bottleneck processing of Task 2 central stages also was found in the second experiment in both age groups. For younger adults, the results provide further evidence falsifying the claim of an immutable response selection bottleneck. For older adults, the evidence suggested that Task 2 affects Task 1 when there is stimulus compatibility but not when there is response compatibility.
\end{abstract}

Alan A. Hartley

ahartley@scrippscollege.edu

1 Department of Psychology, Scripps College, Claremont, CA 91711, USA

2 Laboratoire de Psychologie \& MSHE-Ledoux, Université Bourgogne Franche-Comté, Besançon, France

3 School of Behavioral and Brain Sciences, Center for Vital Longevity, University of Texas-Dallas, Dallas, TX, USA

4 Department of Psychology, Clark University, Worcester, MA, USA
Keywords Aging $\cdot$ Dual task $\cdot$ Psychological refractory period $\cdot$ Response selection $\cdot$ Response-selection bottleneck model $\cdot$ Parallel processing $\cdot$ Backward compatibility effect

Under most circumstances, people cannot perform two tasks at exactly the same time without interference, even when both tasks are very simple. As the temporal overlap between the two tasks increases, the time to perform the second task increases monotonically. Conversely, in many situations for each additional millisecond that the stimulus of the second task is delayed after the onset of the stimulus of the first task (the stimulus onset asynchrony or SOA), the reaction time (RT) to the second task drops by $1 \mathrm{~ms}$. By contrast, the RT to the first task is largely unaffected by SOA. The slowing of the second task at short SOAs often is called the psychological refractory period (PRP) effect by analogy with the time after firing when a neuron is unresponsive to further input (Telford, 1931; Vince, 1948; Welford, 1952). The PRP effect has been replicated in a large number of experiments, thus deserving the status of one of the few laws of contemporary cognitive psychology. Pashler $(1994,1998)$ provides reviews and overviews of the research and theory (also see Spence, 2008; Tombu \& Jolicœur, 2005).

The most successful account of the PRP effect is the response-selection bottleneck (RSB) model, the principal tenet of which is that central processing can be performed for one and only one task at any particular time. Central processing of one of the tasks must be delayed until central processing of the other task is complete. This leads to the prediction that each millisecond by which the arrival of the second task is delayed will be $1 \mathrm{~ms}$ that it will not have to wait for the central processing mechanism to become free. Central processing involves processes of mapping a perceptually identified stimulus onto an abstract program for the action required by the 
situation (Fagot \& Pashler, 1992). These processes are usually identified as response selection, although this is not intended to rule out the involvement of other subprocesses. Even though central processing is viewed as limited and serial; earlier precentral stages (often thought to involve perceptual processes) and later postcentral stages (often thought to involve response-execution processes) are assumed to be more or less unlimited and parallel, although there may be specific input and output interference in sensory registration streams or motor effector streams. It has been demonstrated that versions of capacity-sharing models, in which capacity can be allocated flexibly to each of two tasks, can mimic the predictions of the RSB (Tombu \& Jolicour, 2005). The RSB model is, in fact, a special case of a capacity-sharing model with $100 \%$ of capacity devoted to one task at any one time.

The principal assumption of the RSB model is that there is a bottleneck such that central processing can occur for only one task at a time. Capacity-sharing models allow at least some simultaneous processing. One kind of evidence of simultaneous processing would be a demonstration that manipulations of Task 2-which is presumably blocked by the bottleneck-affected Task-1 processing. In the two experiments reported, we looked for this type of evidence. We then asked whether the evidence was the same or different in younger and older adults.

\section{Experiment 1}

The most reliable demonstrations of parallel processing of two tasks come from PRP experiments in which there is substantial compatibility between the response in Task 1 (R1) and the Task 2 response (R2) (Miller, 2006; Miller \& Alderton, 2006; Thomson, Watter, \& Finkelshtein, 2010; Watter \& Logan, 2006). In the simplest case, Watter and Logan (2006) had R1 and R2 given by the same fingers of the same hand. They observed that R1 was speeded when R2 was identical but that R1 was delayed when R2 was different. The responses need not be identical for such effects to occur. For instance, Hommel (1998) found similar effects when R1 and R2 or R1 and $\mathrm{S} 2$ could be compatible: when R2 was a vocal response, "left" or "right," and R1 was a manual response, left or right key, and also when the color of S2 matched or did not match the color name response given for R1. There is debate about whether such backward compatibility effects are the result of gradually strengthened episodic connections or the result of the connection of R2 and $\mathrm{S} 1$ or R1 by the transient mapping rules held in working memory (Ellenbogen \& Meiran, 2011; Hommel, 1998; Hommel \& Eglau, 2002). Nevertheless, it is clear that there is activation of $\mathrm{R} 1$ by aspects of Task 2, despite the presumption that processing of Task 2 is blocked until the completion of response selection in Task 1. At the same time, the response-selection bottleneck must have been in place still, because the reaction time to Task 2 increased noticeably as SOA shortened in each of the studies mentioned. According to Hommel (1998), parallel response activation occurs at the same time as serial response selection.

We are aware of only two studies that have examined whether backward compatibility effects are affected by advancing age. Grabbe and Allen (2012) explored cross-task compatibility effects using an approach different from PRP. In this study, a color stimulus was first presented and followed 1300 ms later by an auditory cue to give a manual response identifying its color. In the interim, a dot appeared and the participant had to identify the direction of motion of the dot also with a manual response. Responses to both tasks were given with the same fingers of the same hand. In the critical condition, the auditory cue and the moving dot appeared simultaneously (that is, the moving dot appeared $1300 \mathrm{~ms}$ after the color stimulus). When both responses were given by the same finger, there was facilitation; when the responses were given by different fingers, there was inhibition. Younger and older adults showed the same response-response compatibility effects. In the procedure used by Grabbe and Allen, central processing of the first, color task would likely have been completed before the cue to give the response occurred. Thus, the compatibility would have affected only the execution of the response or its monitoring. In recent research Allen, Lien, and Jardin (in press) explored compatibility effects with emotional stimuli using PRP. Task 1 was to identify a facial expression of emotion as happy (positively valenced) or sad (negatively valenced); Task 2 was to identify three different sounds, one positively valenced (laugh), one neutral (cork popping), and one negatively valenced (scream). They found backward compatibility effects for both younger and older adults. RTs on Task 1 were shorter when R1 and R2 were compatible in emotional valence and longer when valence was incompatible.

The purpose of the first experiment reported was to explore age differences and similarities in backward compatibility effects using a method more similar to conventional PRP procedures than that used by Grabbe and Allen (2012). In light of the recent report by Allen et al. (in press), Experiment 1 further explores backward compatibility effects using the PRP procedures similar to those of Hommel (1998). We specifically selected tasks that used different response modalities to reduce any greater output interference that older adults might experience (Hartley, 2001; Hartley \& Maquestiaux, 2007).

\section{Method}

\section{Participants}

Twenty-four younger adults ( 23 females) and 24 older adults (15 females) participated in the experiment. The younger adults averaged 20.33 years of age $(S D=1.28$ years; range, 
$18-21$ years), reported 14.18 years of education $(S D=1.25)$, rated their health at $8.83(S D=1.14)$ on a 10 -point scale with 10 as excellent, and had a median measured visual acuity of 20/25 (range 20/20 to 20/40). The older adults averaged 76.28 years of age $(S D=5.80$; range, $67-87$ years $)$, reported 16.22 years of education $(S D=2.59)$, rated their health at 8.02 $(S D=0.94)$, and had a median measured acuity of $20 / 34$ (range 20/20 to 20/60). All participants had normal color vision, by self-report. All participants received a stipend of 10 USD for participation. Participants were tested at Scripps College.

\section{Tasks}

The experimental procedures were controlled by programs written in E Prime (Version 2.0, Schneider, Eschman, \& Zuccolotto, 2002) running on Intel Pentium computers. Manual responses were made with button presses on a response box (Serial Response Box Model 200a, Psychology Software Tools); vocal responses were sensed by a voiceoperated relay in the response box via a microphone attached to a microphone stand and positioned close to the participant's mouth.

Practice There were three components to the practice task. The first was a color task that introduced what would become the first task in the dual-task trials. On each trial, the letter B appeared in gray to signal that a trial was beginning. It subtended $3.63^{\circ}$ in height by $3.03^{\circ}$ in width at an approximate viewing distance of $60 \mathrm{~cm}$. It was centered on the screen of a 19 in $(48.26 \mathrm{~cm})$ diagonal black display. After $1000 \mathrm{~ms}$, the color changed to red or green. If the color was red, the participant was to press the $\mathrm{z}$ key (labeled "RED") at the left of the keyboard with the left index finger. If the color was green, the participant was to press the/key (labeled "GREEN") at the right of the keyboard with the right index finger. The participant was allowed $3000 \mathrm{~ms}$ to respond. Feedback about correctness was presented for $750 \mathrm{~ms}$. The intertrial interval was $3000 \mathrm{~ms}$. Colors appeared equally often, in random sequence. The second component of the practice introduced a letter task that would become the second task in the dual-task trials. Once again, each trial began with the letter B presented in gray. After $1000 \mathrm{~ms}$, the B was replaced either by the letter "L" or the letter "R," also in gray. If the letter was $L$, the participant was to say "left" into a microphone placed near the lips. If the letter was R, the participant was to say "right." A clock was started when the $\mathrm{L}$ or $\mathrm{R}$ was presented and stopped when the participant's voice was sensed. The participant was allowed $3000 \mathrm{~ms}$ to respond. If the voice was not sensed, a message appeared saying "Could not hear you!" After the response or the error message, the experimenter entered the vocal response given and the participant received feedback about the correctness of the response for $750 \mathrm{~ms}$.
The intertrial interval was $3000 \mathrm{~ms}$. The two letters appeared equally often, in random sequence. The third component of the practice combined the two tasks, namely the color task (Task 1) and the letter task (Task 2), using the PRP procedure. Once again, each trial began with a B in gray for $1000 \mathrm{~ms}$, whereupon the color changed to either red or green. After an SOA of 50, 150, or $650 \mathrm{~ms}$, the B changed to L or R. For each task, $3000 \mathrm{~ms}$ were allowed for a response. Feedback about correctness was provided for each of the responses for $750 \mathrm{~ms}$. The participant was instructed to respond to the color and then the letter and to respond to both tasks as quickly as possible. They were specifically cautioned not to wait to see what the second stimulus was before responding to the first. On half of the trials, the responses for the two tasks were compatible, the color red calling for a left-hand response paired with the letter $\mathrm{L}$ calling for a vocal response of "left" or the color green calling for a right-hand response paired with the letter $\mathrm{R}$ calling for a response of "right." On the other half of the trials, the responses were incompatible.

Dual task The experimental, dual-task trials were identical to those in the third component of practice except that no feedback was provided.

\section{Procedure}

At the outset, informed consent was obtained and demographic information was collected. Visual acuity was measured at the end of the session, using a Snellen chart at $20 \mathrm{ft}(6.11 \mathrm{~m})$. Next were 24 trials in each of the three components of practice. Within each component, stimulus types occurred equally often and were randomly ordered. This was followed by 6 blocks of 24 trials each of the dual task with ad lib rest after each. Each of the six types of trials - compatible and incompatible stimuli combined with SOAs of 50, 150, and $650 \mathrm{~ms}-$ occurred four times in each block. The order of stimuli in a block was random.

\section{Results}

We report analyses performed on data from the experimental dual-task trials; practice trials were not analyzed. Analyses of variance (ANOVAs) were performed on the median reaction time (RT) to Task 1 (the color task), the proportion correct on Task 1, the median RT to Task 2 (the letter task), and the proportion correct on Task 2 as a function of age group (younger or older), response compatibility (Task 1 and Task 2 compatible and incompatible), and SOA (50, 150, and $650 \mathrm{~ms}$ ). Age group was a between-subjects variable and compatibility and SOA were within-subjects variables. Median RTs were used to reduce the influence of outlying observations. In the cases of effects for which Mauchly's test of sphericity was 
significant, the Greenhouse-Geisser correction was applied, and the corresponding probability is reported.

\section{Task 1 (color identification)}

ANOVA on median Task 1 RT showed significant main effects of age group, $F(1,46)=65.49, p<0.001, \eta^{2}=0.59$, of compatibility, $F(1,46)=5.04, p=0.030, \eta^{2}=0.10$, and of SOA, $F(2,92)=9.04, p=0.003, \eta^{2}=0.16$. Older adults $(M=$ $1264 \mathrm{~ms}, S E=60 \mathrm{~ms}$ ) were much slower than younger adults ( $M=578 \mathrm{~ms}, S E=60 \mathrm{~ms})$. Incompatible trials had longer RTs $(M=935 \mathrm{~ms}, S E=44 \mathrm{~ms})$ than did compatible trials $(M=$ $907 \mathrm{~ms}, S E=42 \mathrm{~ms}$ ). Paired comparisons using a Bonferroni correction showed that RTs were shorter at $50 \mathrm{~ms}(M=$ $881 \mathrm{~ms}, S E=37 \mathrm{~ms})$ than at $150 \mathrm{~ms}(M=910 \mathrm{~ms}, S E=$ $40 \mathrm{~ms})$, which were, in turn, shorter than at $650 \mathrm{~ms}(M=$ $973 \mathrm{~ms}, S E=54 \mathrm{~ms}$ ). There also was an interaction of SOA and age group, $F(2,92)=12.13, p=0.001, \eta^{2}=0.21$. The interaction of age with compatibility and SOA approached significance, $F(2,92)=2.76, p=0.072, \eta^{2}=0.06$.

The central questions are whether a backward compatibility effect occurred and, if so, whether it was similar or different for younger and older adults. The answers to these questions are reflected in the three-way interaction, so it was decomposed even though it did not reach conventional significance. Younger adults (Fig. 1a) showed a significant effect of compatibility, $F(1,23)=7.76, p=0.010$, and a significant interaction of compatibility and SOA, $F(2,46)=8.42, p=$ 0.002 . By contrast, older adults (Fig. 1b) showed only a significant effect of SOA, $F(2,46)=14.09, p<0.001$, with RTs increasing monotonically with SOA. T tests were used to compare incompatible and compatible RTs at 50, 150, and $650 \mathrm{~ms}$ SOA for younger and older adults separately. For younger adults, incompatible and compatible RTs differed significantly at $50 \mathrm{~ms}, t(23)=4.42, p<0.001$, with a mean difference of $60 \mathrm{~ms}(S E=14 \mathrm{~ms})$. The difference at $150 \mathrm{~ms}$ was nonsignificant, $t(23)=1.77, p=0.091$, with a mean difference of $22 \mathrm{~ms}(S E=12 \mathrm{~ms})$, as was the difference at $650 \mathrm{~ms}, t(23)=0.26, p=0.80$, with a mean difference of $3 \mathrm{~ms}(S E=13 \mathrm{~ms})$. For older adults, none of the differences approached significance $(p>0.36)$, although at $50 \mathrm{~ms} \mathrm{SOA}$ the difference was $27 \mathrm{~ms}(S E=30 \mathrm{~ms})$. This was not significantly different from the younger adult mean of $60 \mathrm{~ms}, t(46)$ $=1.01, p=0.320$.

ANOVA on the proportion of correct responses to Task 1 showed a main effect of age group, $F(1,46)=4.63, p=0.037$, $\eta^{2}=0.09$, and of compatibility, $F(1,46)=4.23, p=0.045, \eta^{2}=$ 0.08 . Younger adults $(M=0.97, S E=0.01)$ were more accurate than older adults $(M=0.95, S E=0.01)$. Accuracy was higher with compatible stimuli $(M=0.97, S E=0.01)$ than with incompatible stimuli $(M=0.96, S E=0.01)$. There also was a significant interaction of age group and $\mathrm{SOA}, F(2,92)=$ $4.82, p=0.012, \eta^{2}=0.10$. For younger adults, the proportion a Younger Adults

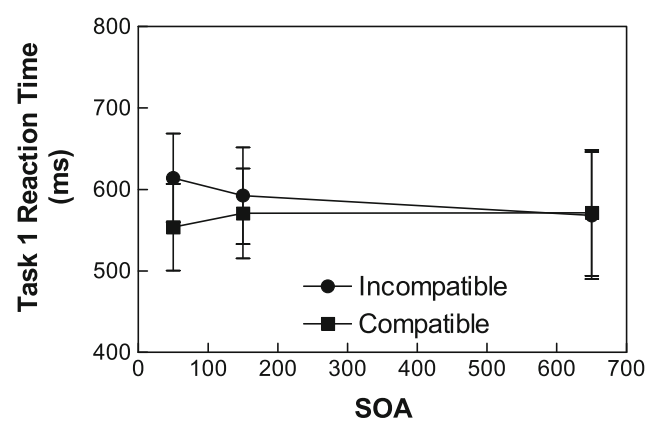

b

Older Adults

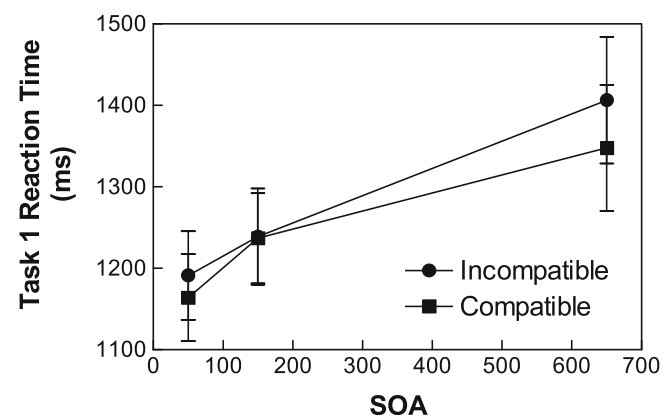

Fig. 1 Task 1 reactions times in Experiment 1 as a function of compatibility between Task 1 and Task 2 and stimulus-onset asynchrony between tasks. Bars show standard errors

correct increased from $50 \mathrm{~ms}(M=0.96, S E=0.01)$ to 150 and $650 \mathrm{~ms}$ (for both $M=0.97, S E=0.01$ ). For older adults, the proportion correct decreased from $50 \mathrm{~ms}(M=0.96, S E=$ 0.01 ) to 150 and $650 \mathrm{~ms}$ (for both $M=0.95, S E=0.01$ ).

\section{Task 2 (letter identification)}

ANOVA of median RTs to Task 2 showed significant main effects of age group, $F(1,46)=17.60, p<0.001, \eta^{2}=0.28$, and of SOA, $F(2,92)=164.69, p<0.001, \eta^{2}=0.78$. RTs for older adults $(M=1273 \mathrm{~ms}, S E=61 \mathrm{~ms})$ were considerably longer than for younger adults $(M=908 \mathrm{~ms}, S E=61 \mathrm{~ms})$. Paired comparisons for SOA showed a classic PRP effect with RTs longer at $50 \mathrm{~ms}(M=1226 \mathrm{~ms}, S E=46 \mathrm{~ms})$ than at $150 \mathrm{~ms}(M=1158 \mathrm{~ms}, S E=46 \mathrm{~ms})$, which were longer than at $650 \mathrm{~ms}(M=888 \mathrm{~ms}, S E=42 \mathrm{~ms})$. The interaction of age group and SOA was not significant, $F(2,92)=2.69, p=0.097$, $\eta^{2}=0.06$. The PRP effect $\left(R T_{50}-R T_{650}\right)$ was numerically smaller in the older adults $(M=292 \mathrm{~ms})$ than in the younger adults $(M=383 \mathrm{~ms})$. There were significant interactions of compatibility and SOA, $F(2,92)=6.81, p=0.002, \eta^{2}=0.13$, and of age group with compatibility and $\operatorname{SOA}, F(2,92)=3.79$, $p=0.027, \eta^{2}=0.08$. The three-way interaction is shown in Fig. 2. Decomposition of the three-way interaction showed, for the younger adults, significant effects of compatibility, $F(1,46)=9.67, p=0.005$, SOA, $F(2,92)=154.01, p<$ 0.001 , and of the interaction of compatibility and SOA, $F(2$, 


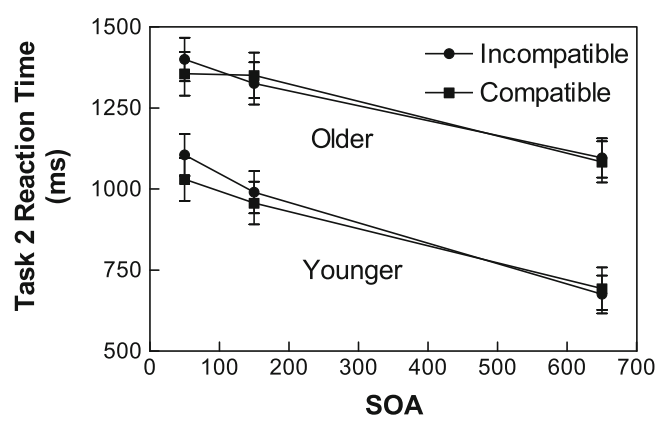

Fig. 2 Task 2 reaction times in Experiment 1 as a function of age group, compatibility between Task 1 and Task 2, and stimulus-onset asynchrony between tasks. Bars show standard errors

$92)=12.07, p<0.001$. For the older adults, the only significant effect was SOA, $F(1,46)=48.37, p<0.001$. Comparisons of incompatible and compatible RTs at each SOA for the younger adults showed that the difference was significant at $50 \mathrm{~ms}$, with a mean difference of $75 \mathrm{~ms}(S E=$ $18 \mathrm{~ms}), t(23)=4.25, p<0.001$, and at $150 \mathrm{~ms}$, with a mean difference of $34 \mathrm{~ms}(S E=14 \mathrm{~ms}), t(23)=2.44, p=0.023$, but not at $650 \mathrm{~ms}$, with a mean difference of $-18 \mathrm{~ms}(S E=12 \mathrm{~ms})$, $t(23)=-1.53, p=0.14$. There were no significant differences for older adults.

Analysis of the proportion correct on Task 2 showed only a significant main effect of age group, $F(1,46)=25.88, p<$ $0.001, \eta^{2}=0.36$, with accuracy slightly higher for younger adults $(M=0.995, S E=0.002)$ than for older adults $(M=$ $0.980, S E=0.002)$.

\section{Discussion}

For the younger adults, there were clear backward compatibility effects. At the shortest SOA, $50 \mathrm{~ms}$, compatibility of the Task 1 response with the Task 2 response shortened Task-1 RTs relative to incompatible conditions. There was a smaller, nonsignificant effect at $150 \mathrm{~ms}$ SOA and almost no effect at the longest SOA, $650 \mathrm{~ms}$. The RSB model predicts that any manipulation that affects Task 1 precentral or central processing - such as the compatibility effects here-will carry over onto the RT to Task 2 (see Pashler, 1994, Principle 1). If a manipulation adds, for example, $50 \mathrm{~ms}$ to the processing of Task 1, then Task 2 also must wait those additional $50 \mathrm{~ms}$ at short SOAs. At long SOAs, however, Task 2 does not have to wait for the bottleneck to open, because Task 1 central processing would be complete and the Task 1 manipulation should have no effect on Task 2. The result of the Task 1 carryover would be an overadditive interaction in Task 2 RT such that the effects are greatest at sort SOAs. Such a carryover was observed in the younger adults. The mean difference between compatible and incompatible RTs on Task 1 was $75 \mathrm{~ms}$ at $50 \mathrm{~ms} \mathrm{SOA}$; the difference for Task 2 was $60 \mathrm{~ms}$.

The pattern of results for older adults was unclear. The difference between compatible and incompatible conditions at the shortest SOA $(27 \mathrm{~ms})$ did not differ significantly from zero. Nevertheless, it was not significantly different from the difference for younger adults $(60 \mathrm{~ms})$. There may be no backward compatibility effect for older adults, or there may be a real effect but one that is smaller than that observed in younger adults with the reliability of the effects obscured by relatively small sample sizes and large variability. We will return to this issue shortly. Without a significant backward compatibility effect, there was no reason to expect a carryover effect onto Task 2 RTs, and none was seen. There was a PRP effect with RTs to Task 2 at $50 \mathrm{~ms}$ SOA slower by $292 \mathrm{~ms}$ than at $650 \mathrm{~ms}$. There were two anomalous features to the results for the older adults. First, Task 1 RTs increased by $199 \mathrm{~ms}$ from $50 \mathrm{~ms}$ SOA to $650 \mathrm{~ms} \mathrm{SOA}$. For younger adults, Task 1 RTs were unaffected by SOA, averaging across compatible and incompatible conditions. For older adults, it may have been disruptive for the second stimulus to arrive about the time execution of the first response was commencing. Second, the PRP effect was actually smaller in older adults (292 ms) than in younger adults $(383 \mathrm{~ms})$. The average reaction time for older adults with an SOA of $650 \mathrm{~ms}$ was $1427 \mathrm{~ms}$. Thus, on many trials, participants would still have been actively engaged in processing Task 1 at the onset of Task 2. This would lead to a substantial underestimation of the true PRP effect in older adults.

The slowing of Task-1 RTs with increasing SOA and the absence of compatibility effects in the older adults could reflect response grouping, in which the response to the first task is delayed until the second task is processed. We identified seven older adults who could have been grouping responses based on very short inter-response intervals and on frequent instances of giving the response to Task 2 before that to Task 1. We determined IRI as the difference between the time stamp for R2 and the time stamp for R1. Mean IRIs less than $150 \mathrm{~ms}$ were considered very short and negative mean IRIs were considered to indicate reversed responding. We repeated the analyses of Task 1 RTs with these individuals removed. In the reanalysis, there were significant main effects of age group, $F(1,39)=46.78, p<0.001, \eta^{2}=0.54$, compatibility, $F(1,39)=$ 5.26, $p=0.027, \eta^{2}=0.12$, and SOA, $F(2,78)=3.37, p=$ $0.050, \eta^{2}=0.08$. There were significant interactions of age group and SOA, $F(2,78)=4.28, p=0.027, \eta^{2}=0.10$, and of age group, compatibility, and SOA, $F(2,78)=4.40, p=0.019$, $\eta^{2}=0.10$. Testing whether the observed backward compatibility effects $\left(\mathrm{RT}_{\text {INCOMPATIBLE }}-\mathrm{RT}_{\text {COMPATIBLE }}\right.$ ) were significantly greater than zero, the results for younger adults were, of course, unchanged from the original analysis. For the older adults, the differences from zero were nonsignificant at $50 \mathrm{~ms}$ $\operatorname{SOA}(M=-13 \mathrm{~ms}, S E=31 \mathrm{~ms}), 150 \mathrm{~ms} \mathrm{SOA}(M=-21 \mathrm{~ms}$, $S E=43 \mathrm{~ms})$, and $650 \mathrm{~ms} \mathrm{SOA}(M=26 \mathrm{~ms}, S E=35 \mathrm{~ms})$. Comparing the compatibility effects for younger and older adults, they were significantly greater for younger adults than for older adults at $50 \mathrm{~ms} \mathrm{SOA}, t(39)=2.41, p=0.021$, but not at $150 \mathrm{~ms} \mathrm{SOA}, t(39)=1.09, p=0.091$, or $650 \mathrm{~ms} \mathrm{SOA}, t(39)$ 
$=-0.90, p=0.799$. In Task 2, the pattern of results was unchanged. With response grouping taken into account, the results are consistent with the interpretation that a backward compatibility effect was found in the younger adults but not in the older adults.

Grabbe and Allen (2012) and Allen et al. (in press) found backward compatibility effects of equivalent magnitude in younger and older adults. We did not find a backward compatibility effect in older adults. We did find it in younger adults, contrary to any claim that our manipulation was simply too weak to produce an effect. A simple but not helpful explanation for the difference in findings is that older adults show backward compatibility effects in some situations but not in others. Grabbe and Allen may have seen the effects of residual motor effector activation as the same fingers of the same hand were used for responding to the two tasks. The stimuli used by Allen et al. which have naturally meaningful emotional significance may have evoked parallel processing, whereas the stimuli used here, which are given meaning only by experimental instructions, did not. These are not parsimonious explanations. That will have to await the accumulation of more evidence about when older adults show backward compatibility effects and when they do not.

\section{Experiment 2}

In Experiment 1, we found evidence of a backward compatibility effect between responses in younger adults but not in older adults. In Experiment 2, we explored compatibility between the stimuli for the two tasks, as did Grabbe and Allen (2012). We made use of the Stroop (1935) effect, which evidences very strong, automatic associations between color names and colored stimuli. Aside from a general slowing, there are no age-related differences in the Stroop effect (Verhaeghen, 2014). In Experiment 2, Task 1 was to identify the color of a patch by saying the color name. The stimuli for Task 2 were color names that were either congruent or incongruent with the Task 1 color, or they were color-irrelevant, neutral words. To simplify responding, the actual task was to identify by a button press the first letter of the word. We expected that the Task 2 words would be read automatically as soon as they appeared (Wheeler, 1970). Concerning Task 1, we expected that with a short SOA (a high task overlap), the color word would have a backward compatibility effect on the naming of the color. Task-1 color naming should be speeded by congruent Task- 2 color words but should be slowed by incongruent Task-2 color words. If automaticity of reading is stronger in older than younger adults, we might expect to see larger effects in the older adults.

As a result of Experiment 1, we expanded the instructions not to group responses. We increased the number of SOAs to allow a more fine-grained analysis of compatibility effects. The amount of practice also was increased.

\section{Method}

\section{Participants}

The participants were 37 undergraduate younger-adult volunteers ( 23 females) and 24 older-adult volunteers ( 12 females) from the local community. The younger adults averaged 19.71 years of age $(S D=1.15$ years); they reported an average of 13.86 years of education ( $S D=0.88$ years); the mean rating of health was $8.38(S D=1.35)$ on a 10 -point scale $(10$ was excellent); their median Snellen visual acuity was 20/22 (range 20/15 to 20/40). The older adults averaged 76.54 years of age ( $S D=7.80$ years); they reported an average of 16.81 years of education $(S D=2.68$ years); they rated their health $8.38(S D=1.29)$ on the 10-point scale; their median Snellen visual acuity was 20/34 (range, 20/20 to 20/60). All participants received a stipend of 15 USD. They were screened for color blindness by self-report. Data from two other individuals reporting color blindness were not included in the analysis. Participants were tested at Scripps College.

\section{Tasks}

Task 1 (color identification) Each trial in the color identification task began with a dark screen with a white outline rectangle at the center of the display, presented for $1,000 \mathrm{~ms}$. At an approximate viewing distance of $60 \mathrm{~cm}$, the outline rectangle subtended $16.70^{\circ}$ in width by $5.24^{\circ}$ in height. After a fixed foreperiod of $1000 \mathrm{~ms}$, the white outline rectangle was filled with a color that was red, green, blue, or purple (the background remained dark). The participant was instructed to respond as quickly and accurately as possible by speaking the name of the color. Latency was determined from the onset of the color until a vocal response was sensed. After the verbal response was sensed, at the end of the trial, the participant was probed to identify the verbal response that had been given by pressing a key on the computer keyboard with the left hand. If no voice response was sensed, the message "No voice response detected" was displayed for $750 \mathrm{~ms}$. If a response was sensed, the next screen provided feedback about the correctness of the reported response for $1,000 \mathrm{~ms}$. The colors were randomized across trials.

Task 2 (letter identification) Each trial in the letter identification task began with a dark screen with a rectangle, $16.70^{\circ}$ by $5.24^{\circ}$, outlined in white and filled with gray, at the center of the display, for 1,000 ms. After the fixed foreperiod, a word in white was superimposed on the gray outline rectangle. The word was either a word drawn from a set of color words 
(red, green, blue, purple) or from a set of color-unrelated words (rap, grasp, bolt, pursue). The participant was instructed to respond as quickly and accurately as possible by pressing a button on a response box corresponding to the first letter of the word, $r, g$, or $p$ (using fingers of their right hand). Labels were placed above the buttons. Following the response, the correctness of the response was signaled for 1 , $000 \mathrm{~ms}$. The words were randomized across trials.

Dual task Each dual-task trial began with a dark screen with a white outline rectangle, $16.70^{\circ}$ by $5.24^{\circ}$, presented at the center of the display for $1,000 \mathrm{~ms}$. After the fixed foreperiod, the outline rectangle was filled with one of the colors used in the Color Identification task. After a stimulus-onset asynchrony (SOA) randomly drawn from the set of 50, 100, 150, 200, 400, 600 , and $1000 \mathrm{~ms}$, one of the words from the Letter Identification task was superimposed, in white, on the colored rectangle. The participant was instructed to respond verbally to the color as in the Color Identification task and manually to the first letter of the word as in the Letter Identification task. The instructions were to respond as quickly as possible, first to the color and then to the letter, but without sacrificing accuracy and not to group responses. No feedback was provided. For each task, $3000 \mathrm{~ms}$ from the onset of the stimulus was allowed for a response. The combinations of color, SOA, and word were randomized across trials, with the result that the words could be categorized as color words that were congruent with the color of the filled rectangle, color words that were incongruent, or non-color words unrelated to the color of the filled rectangle (neutral condition).

\section{Procedure}

After the tasks were explained, the participant first completed a practice session comprising three blocks with feedback on each trial: 48 trials of the Color Identification task only, 48 trials of the Letter Identification task only, and 48 dual task trials. Next, the participant completed six blocks of 48 experimental dual task trials without feedback, with ad lib rest after each block. The intertrial interval throughout was 1,000 ms. Information for year of birth, education, and self-rated health status was collected, and Snellen visual acuity was measured after the experimental blocks.

\section{Results}

Analyses of variance (ANOVAs) were performed on median dual task RTs for both tasks for trials on which both responses were correct and on the proportion correct. Age group (younger or older) was a between-subjects variable; word type (congruent, incongruent, and unrelated) and $\operatorname{SOA}(50,100,150$, 200, 400, 600, and $1000 \mathrm{~ms}$ ) were within-subjects variables.
Task 1 (color identification) Means of the median Task 1 RTs are shown in Fig. 3a for each word type and SOA for each age group. There were significant main effects of age group, $F(1$, $59)=21.39, p<0.001, \eta^{2}=0.27$, and word type, $F(2,118)=$ 12.84, $p=0.001, \eta^{2}=0.18$. RTs were shorter for younger adults $(M=761 \mathrm{~ms}, S E=44 \mathrm{~ms})$ than for older adults $(M=$ $1085 \mathrm{~ms}, S E=55 \mathrm{~ms})$. RTs were slowest when the Task 2 word was incongruent with the color on Task $1(M=955 \mathrm{~ms}$, $S E=36 \mathrm{~ms})$, intermediate when the word was color-neutral $(M=920 \mathrm{~ms}, S E=35 \mathrm{~ms})$, and fastest when the word was congruent with the color $(M=894 \mathrm{~ms}, S E=36 \mathrm{~ms})$. Color identification RT was not affected by SOA, $F(6,354)=0.90$, $p=0.492, \eta^{2}=0.02$. The interaction of word type and SOA approached significance, $F(12,708)=1.91, p=0.084, \eta^{2}=$ 0.03 . Examination of the simple main effects of word type at each SOA showed significant effects at SOAs of 50, 100, 150 and $200 \mathrm{~ms}(F \mathrm{~s}$ with $d f=2,118$, respectively, 14.29, 6.60, $6.96,5.10 ; p s<0.008)$. Effects were not significant for SOAs of 400,600 , or $1000\left(F_{\mathrm{S}}<1\right)$. The interaction of age group with word type and SOA did not approach significance, $F(14$, $826)=1.06, p=0.376, \eta^{2}=0.02$. Consistent with the nonsignificant three-way interaction, the mean difference between incongruent and congruent conditions for SOAs from 50 to $200 \mathrm{~ms}$ was comparable: $92 \mathrm{~ms}$ for the younger adults and $107 \mathrm{~ms}$ for the older adults.

Task 2 (letter identification) Mean RTs are shown in Fig. $3 b$. ANOVA with the same factors as for the analysis of the Color Identification task showed significant main effects of age group, $F(1,59)=80.75, p<0.001, \eta^{2}=0.58$, word type, $F(2,118)=74.60, p<0.001, \eta^{2}=0.56$, and SOA, $F(7,354)$ $=167.32, p<0.001, \eta^{2}=0.74$. Younger adults $(M=1158 \mathrm{~ms}$, $S E=56 \mathrm{~ms})$ were overall faster than older adults $(M=$ $1965 \mathrm{~ms}, S E=70 \mathrm{~ms})$. Color-congruent trials $(M=$ $1455 \mathrm{~ms}, S E=45 \mathrm{~ms}$ ) were faster than color-neutral trials $(M=1579 \mathrm{~ms}, S E=46 \mathrm{~ms})$, which were faster than colorincongruent trials $(M=1650, S E=46 \mathrm{~ms})$. RTs decreased monotonically with SOA from $50 \mathrm{~ms}(M=1803 \mathrm{~ms}, S E=$ $47 \mathrm{~ms})$ to $1000 \mathrm{~ms}(M=1241 \mathrm{~ms}, S E=44 \mathrm{~ms})$, a PRP effect $\left(R T_{50}-R T_{1000}\right)$ of $562 \mathrm{~ms}$. The interaction of age group and SOA was not significant, $F(6,354)=1.56, p=0.158, \eta^{2}=$ 0.03 . The PRP effect was numerically but not statistically larger for younger adults $(M=584 \mathrm{~ms})$ than for older adults $(M=540 \mathrm{~ms})$. Because the mean Task $1 \mathrm{RT}$ for older adults was longer than the longest SOA, the PRP effect might be underestimated. There was a significant interaction of word type and SOA, $F(12,708)=2.58, p=0.002, \eta^{2}=0.04$. To explore this interaction, we calculated the simple main effect of word type at each SOA. These were significant from $50 \mathrm{~ms}$ SOA through $400 \mathrm{~ms}$ SOA with the largest $F_{\mathrm{s}}(2,118)$ of 25.02 at $50 \mathrm{~ms} \mathrm{SOA}$ and 32.75 at $100 \mathrm{~ms}$ SOA. From a different perspective, the difference between incongruent and congruent RTs (RT INCONGRUENT - RT CONGRUENT) was 
a

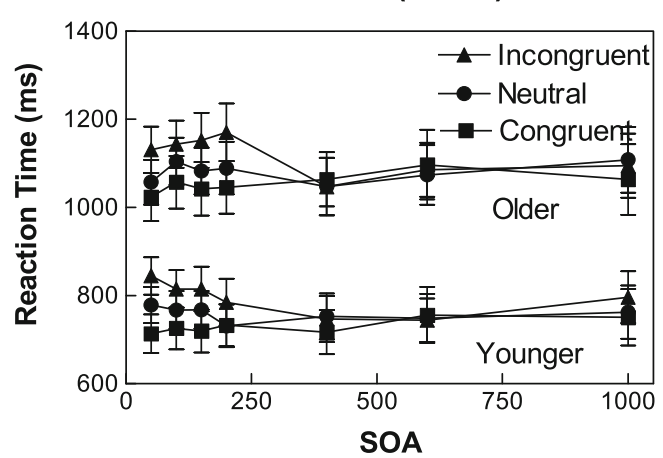

b

Task 2 (Letter)

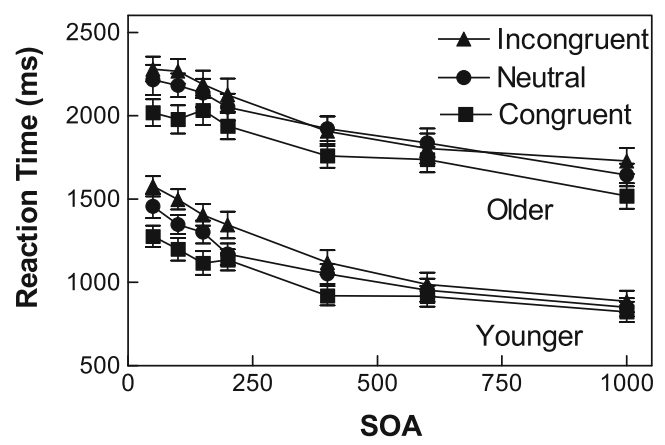

Fig. 3 Reaction times to color Task 1 (panel a) and the letter Task 2 (panel b) in Experiment 2 as a function of age group, congruency between Task 1 and Task 2, and stimulus-onset asynchrony. Bars show standard errors

larger for SOAs of 50 (281 ms), $100(293 \mathrm{~ms}), 150(222 \mathrm{~ms})$, and $200(197 \mathrm{~ms})$ than for SOAs of $600(69 \mathrm{~ms})$ and 1000 (136 ms). The interaction of word type and SOA with age group was not significant, $F(12,708)=0.76, p=0.694, \eta^{2}$ $=0.01$.

Proportion correct ANOVA on the proportion of correct responses in the Color Identification task (Task 1) produced a significant effect of age group, $F(1,60)=9.51, p=0.003, \eta^{2}=$ 0.14 , with younger adults significantly more likely to respond correctly $(M=0.99, S E=0.01)$ than older adults $(M=0.93, S E$ $=0.02)$. ANOVA on the proportion of correct responses in the Letter Identification task (Task 2) showed a significant main effect of word type, $F(2,120)=23.40, p<0.001, \eta^{2}=0.28$, with accuracy higher for color-congruent trials $(M=0.99, S E$ $=0.002)$ and for color-neutral trials $(M=0.98, S E=0.004)$ than for color-incongruent trials $(M=0.95, S E=0.008)$. Although the main effect of age group was not significant, $F(1,60)=3.47, p=0.538, \eta^{2}=0.05$, there was a significant interaction of age group and word type, $F(2,120)=6.19, p=$ $0.011, \eta^{2}=0.09$. The interaction occurred because older adults $(M=0.93, S E=0.02)$ were significantly less accurate than younger adults $(M=0.97, S E=0.01)$ for color-incongruent trials, whereas there were no age differences for colorcongruent or color-unrelated words.
Task 1 carryover

As we noted in the Discussion of Experiment 1, the RSB model predicts that any manipulation that affects Task 1 precentral or central processing - such as the Stroop effects - will carry over onto the RT to Task 2 . The question is whether at short SOAs, the effects of color-word congruency on Task 2 were any larger than what would be predicted by the carryover from Task 1, that is whether the overadditive interaction is larger than would be expected from the carryover of the congruency effect from Task 1 alone. To address this question, we calculated the effects for congruent combinations (congruency effect, $\mathrm{RT}_{\mathrm{NEUTRAL}}-\mathrm{RT}_{\mathrm{CONGRUENT}}$ ) and for incongruent combinations (incongruency effect, $\mathrm{RT}_{\text {INCONGRUENT }}-\mathrm{RT}_{\text {NEUTRAL }}$ ) both for Task 1 and Task 2 for SOAs from 50 to $200 \mathrm{~ms}$ (i.e., the SOAs for which the average congruency effects were large). We then performed an ANOVA on the difference between the Task 2 and Task 1 effects (see Thomson \& Watter, 2013, for a similar approach). There were no significant effects: There was no effect of age group, type of effect (congruency or incongruency), or SOA, and no interactions of those factors. The overall difference $(M=74 \mathrm{~ms}, S E=10 \mathrm{~ms})$, however, was significantly greater than zero, $F(1,59)=54.69, p<0.001$. That is, the Task 2 congruency and incongruency effects were significantly larger than would be predicted from Task 1 carryover alone.

\section{Discussion}

\section{Effects on task 1}

In contrast to Experiment 1 in which younger but not older adults showed backward response compatibility effects, in Experiment 2 both age groups showed backward stimulus compatibility effects, effects that did not differ in size. This shows that reading the words comprising the Task 2 stimulus could proceed in parallel with Task 1 processing. The finding is at odds with that of Grabbe and Allen (2012) who did not find stimulus compatibility effects, a fact to which we will return in the General Discussion.

\section{Effects on task 2}

There was an interaction of congruity effects with SOA in Task 2 RTs. Moreover, the effects were larger than would be predicted if they were simply the result of carryover of the effects of congruity on Task 1 . This raises the question of whether the larger effects might reflect the presence of simultaneous processing, processing of the central stage of Task 2 before central processing of Task 1 was complete. To put this possibility in perspective, we need to review evidence suggestive of simultaneous processing. 


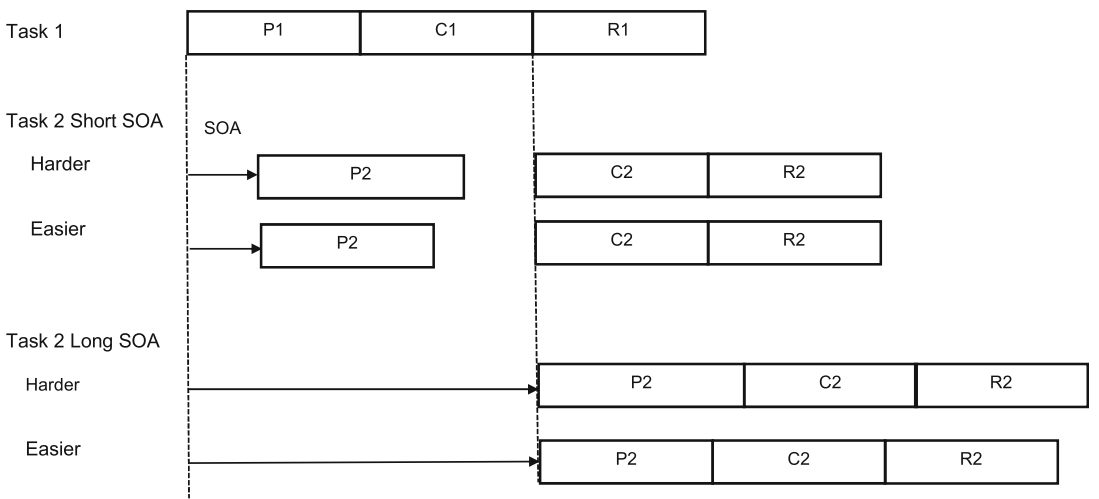

Fig. 4 Case 1: Aspects of Task 2 processing that escape the bottleneck are perceptual processes. SOA, Stimulus Onset Asynchrony; Pn, Precentral stage of Task n; Cn, Central processing stage of Task n; Rn, postcentral Response execution stage of Task $n$. Time on a trial runs from left to right

One class of situations in which some have argued that simultaneous processing or at least partial bottleneck bypass is possible is that of tasks involving word reading, such as phoneme judgment, word recognition, and lexical decision tasks (Allen et al., 2002; Cleland, Gaskell, Quinlan, \& Tamminen, 2006; Gaskell, Quinlan, Tamminen, \& Cleland, 2008; Lien et al., 2006; Rabovsky, Álvarez, Hohlfeld, \& Sommer, 2008). For most adults, reading is an easy and extremely well-practiced task, so much so that it might be thought of as nearly reflexive or automatic. The hundreds of demonstrations of the Stroop (1935) phenomenon, in which the presence of an irrelevant color word facilitates or interferes with the naming of a displayed color, confirm this (MacLeod, 1991). If the second of two tasks involves or can be facilitated by reading, can central processing on that task begin before central processing on the first task is complete or must it wait until the central bottleneck has cleared? To address this question, McCann, Remington, and Van Selst (2000) gave a pitch discrimination as the first task and a lexical decision or word naming task as the second task with SOAs between the tasks of 100 to $800 \mathrm{~ms}$. High-frequency words were responded to more quickly than low-frequency words, but this slowing combined additively with the slowing due to decreasing
SOA. They interpreted this result to mean that the effect of word frequency, and therefore the effects of visual word processing generally, must come after the RSB (see also Pashler, 1994, Principle 4).

Subsequent experiments have obtained different results (Allen et al., 2002; Cleland et al., 2006; Gaskell et al., 2008; Lien et al., 2006; Rabovsky et al., 2008), reporting instead that the effects due to word reading are smaller at very short SOAs than they are at long SOAs, at which there should be little interference between the tasks. For example, in a lexical decision task, high- and low-frequency words are identified as words faster than nonsense strings are identified as nonwords, but the difference between high- and low-frequency words was smaller at short SOAs than at long SOAs (Allen et al., 2002; Cleland et al., 2006; Lien et al., 2006; Rabovsky et al., 2008). That is, there was an underadditive interaction with RTs for high- and low-frequency words converging with decreasing SOA. Ruthruff, Allen, Lien, and Grabbe (2008) found that good readers showed this interaction whereas poor readers did not. McCann et al. (2000) had found some evidence for underaddivity but discounted it. The RSB model postulates three stages: an early, precentral perceptual stage (labeled P in Figs. 4 and 5), a central-processing stage (C),

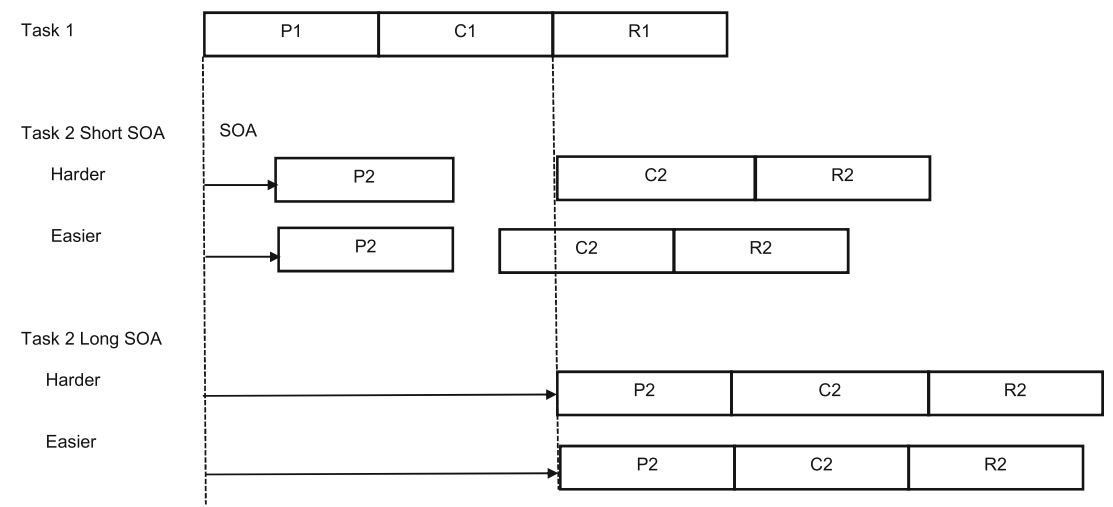

Fig. 5 Case 2: Aspects of Task 2 processing that escape the bottleneck are central processes. SOA, Stimulus Onset Asynchrony; Pn, Precentral stage of Task n; Cn, Central processing stage of Task n; Rn, postcentral Response execution stage of Task n. Time on a trial runs from left to right 
and a late, postcentral response-execution stage (R). Underadditive effects are a signature in the RSB model of Task 2 precentral, perceptual processes being performed in parallel with Task 1 precentral or central processing (see Pashler, 1994, Principle 3). It is a correct inference that the presence of an underadditive interaction means that some aspects of reading of the word (Task 2) must have taken place at the same time as the precentral or central stages of processing of Task 1 . Some aspects of word reading must be automatic in the sense that they are not subject to the capacity limitations imposed by the central response-selection bottleneck. Are those central aspects of Task 2 processing or are they precentral?

If we suppose that some aspects of a task such as word reading can avoid the bottleneck, then there are two possibilities or cases. Case 1 is that those aspects are part of perceptual processing; Case 2, which we will describe next, is that those aspects are part of central processing. The model for Case 1 is shown in Fig. 4. Our example concerns low-frequency and high-frequency words, but the conclusions generalize to any situation in which there are harder and easier conditions. For consistency, we have called the two conditions harder and easier in Figs. 4, 5, and 6. The predicted results from this model are shown in the left panel of Fig. 6. Because of the cognitive slack (McCann \& Johnston, 1992), while Task 2 is waiting for the central processor to become available, the effect of word frequency is wholly or partially absorbed at short SOAs but remains at long SOAs. This is seen as an underadditive interaction of word frequency and SOA with the differences between harder and easier conditions narrowing as SOA decreases. This is precisely what was seen in the experiments summarized above.

An alternative scenario is that central processing in the two tasks can overlap, which we call Case 2. The model for Case 2 in which the aspects of processing that can escape the bottleneck are part of central processing is shown in Fig. 5. Central processing of Task 2 begins before central processing of Task 1 is complete and processing of the easier condition (e.g., congruent conditions) begins earlier than that of the harder condition (e.g., incongruent conditions). The predicted results for this model are shown in the right panel of Fig. 6. The overadditive interaction is such that the difference between harder and easier conditions increases with decreasing SOA. There is evidence consistent with Case 2. Pashler, Carrier, and Hoffman (1993) found that when Task 2 required a saccade to a peripherally appearing target, PRP effects were greatly reduced relative to a condition in which a central cue indicated the location of a peripheral target. Hartley, Seaman, and Maquestiaux (2015) confirmed this finding and showed that it was true for older adults as well. Hartley et al. further showed the same result in both age groups when Task 2 required a body tilt in the direction of a peripheral target. In each of these experiments, there was still a small but significant PRP effect, indicating that some part of Task 2 processing still proceeded serially. The procedures of Pashler et al. and Hartley et al. arguably involved some natural connection between the Task 2 stimulus and response-what Greenwald (1972; Greenwald \& Shulman, 1973) has termed ideomotor compatibility. Ideomotor compatibility is a special case of stimulus-response compatibility in which "a stimulus corresponds to sensory feedback from its required response" (Greenwald, 1972, p. 52): for example, when the required response is to say "right" in response to the auditorily presented stimulus "right."

There has been considerable debate about whether ideomotor compatible tasks can completely bypass the responseselection bottleneck, leading to apparently perfectly parallel processing (Greenwald, 2003, 2004, 2005; Lien, Proctor, and Allen, 2002; Lien, Proctor, \& Ruthruff, 2003; Lien, McCann, Ruthruff, \& Proctor, 2005), but there is no disagreement that they result in smaller PRP effects than non-ideomotor compatible tasks. The important point is that in Experiment 2, Task 2 stimuli and responses were not ideomotor compatible. There is no natural connection between a word and pressing an arbitrarily-assigned key to identify the first letter.

Lien et al. (2005) proposed a model equivalent to Case 2, the engage-bottleneck-later model, in which an early substage of response selection can proceed before the bottleneck is released. This model was an attempt to explain PRP effects that were smaller than predicted by the standard response
Case 1

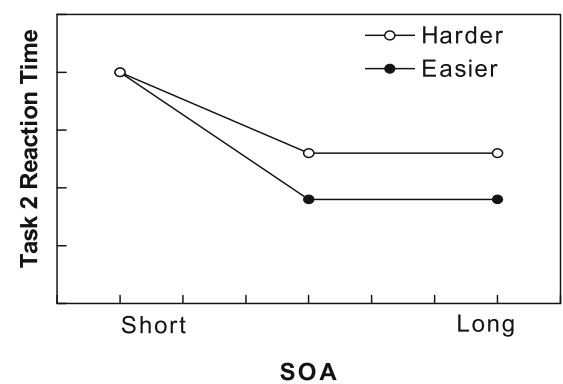

Case 2

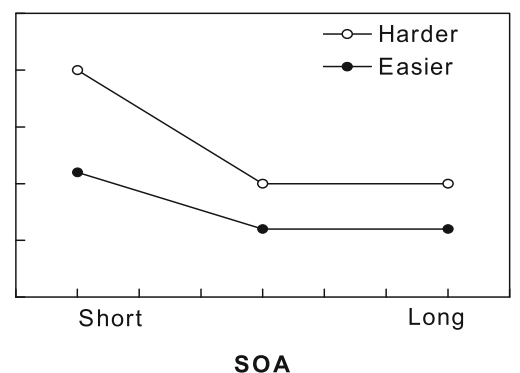

Fig. 6 Predicted results for harder (e.g., lexical decisions for low-frequency words) and easier (e.g., high-frequency words) conditions from Case 1 (processes avoiding the bottleneck are perceptual) and from Case 2 (processes avoiding the bottleneck are central) 
selection bottleneck model when the stimuli and responses on Task 2 were ideomotor compatible.

We can conclude from this review that the results for Task 2 RTs in Experiment 2 were consistent with Case 2, but not with Case 1. This would lead us to conclude that central processing in Task 2 was not fully postponed until central processing of Task 1 was complete. There is, however, a competing explanation. Suppose that central processing of Task 2 is fully postponed until central processing of Task 1 is complete. Suppose also, though, that activation of R1 persists for a short time. At short SOAs, the argument goes the activation of R1 could lead directly to the activation of R2, even though central processing was completely separate. A difficulty with this explanation is that R1 is to name a color, whereas R2 is quite different: to press a key corresponding to the first letter of $\mathrm{S} 2$. However, we could assume that the activation of R1 speeds or slows the processing of the word and that affects the response selection for the letter. In fact, that would mean that the activation of R1 was affecting central processing - response selection-of Task 2. Our tentative conclusion is that the results for Task 2 in Experiment 2 were consistent with some simultaneous central processing of the two tasks. It is important to emphasize that the only evidence for parallel processing is that the effect of congruity was $74 \mathrm{~ms}$ larger than predicted by carryover from Task 1. This one piece of possible evidence for partial bypass of the response-selection bottleneck is very different from the apparently complete bypass seen in younger adults given very extensive training (Maquestiaux, Laguë-Beauvais, Ruthruff, \& Bherer, 2008; Ruthruff, Van Selst, Johnston, \& Remington, 2006).

\section{General discussion}

\section{Backward compatibility effects}

We found backward response-compatibility effects in younger but not older adults in Experiment 1. We found similar backward stimulus-compatibility effects in both age groups in Experiment 2. Grabbe and Allen (2012) and Allen et al. (in press) both found backward compatibility effects in older adults with manipulations of the compatibility of R2 and R1. As we have noted, understanding the source of the difference in results will require further research.

In Experiment 2 of Grabbe and Allen (2012) S2-a dot whose direction of movement had to be discriminated - was either in the same color as S1 (compatible stimulus) or a different color (incompatible stimulus). Task 1 was to discriminate the color of the stimulus. However, participants had $1300 \mathrm{~ms}$ in which to process Task 1 before the onset of S2. Quite possibly R1 was fully selected and loaded for execution before the arrival of S2 so that competing information might have no effect. The procedures of their Experiment 2 are not readily comparable with those of our Experiment 2, so differences in results should not be surprising. Again, at this point we can say that older adults show backward stimuluscompatibility effects in some but not all situations in which younger adults show them.

Effects that slow processing are typically larger in older adults. For example, in a meta-analysis, Verhaeghen (2014) found that the average incongruity effect in the Stroop task $\left(\mathrm{RT}_{\text {INCONGRUENT }}-\mathrm{RT}_{\text {NEUTRAL }}\right)$ was $254 \mathrm{~ms}$ for younger adults but $479 \mathrm{~ms}$ for older adults. Older adults are slower overall, so one might expect such effects to be amplified proportionally. Verhaeghen showed, however, that when baseline (neutral condition) slowing of older adults was taken into account, age differences vanished. Because older adults were much slower in these experiments, that makes it particularly striking that the backward compatibility effects were not only not larger in older adults but were in fact similar (Experiment 2: younger adult mean effect $=92 \mathrm{~ms}$; older adult, $107 \mathrm{~ms}$ ) or smaller (Experiment 1: younger adult mean effect $=60 \mathrm{~ms}$; older adult, $6 \mathrm{~ms})$.

\section{Parallel processing}

An anomalous finding in both of the present experiments was that the PRP effect was not significantly different in older and younger adults (in fact it was numerically smaller in older adults in Experiment 1). In most reports, the PRP effect is significantly larger in older adults (Hartley \& Little, 1999) as in the Stroop effect, but this is not always the case (Allen et al., in press; Hartley, 2001, manual-verbal responses; Hartley \& Little, 1999, Experiments 3 and 6; Hartley et al., 2015) One likely possibility is that the size of the PRP effect was underestimated for older adults. Because mean RT to Task 1 was greater than the longest SOA, on many trials at the longest SOA participants would still have been processing Task 1 when the S2 arrived and Task 2 processing would be postponed. A second possibility is that in the present experiments, older adults were engaging in more parallel processing. Because their RTs to Task 1 were very long, this may have allowed more time to carry out processing of Task 2 at the same time. Note that this need not imply they were carrying out central processing of the two tasks at the same time. The RSB model countenances precentral processing of Task 2 while Task 1 is being processed, what we called Case 1 . Parallel processing of this sort could reduce the size of the PRP effect.

Allen et al. (2002) followed by Lien et al. (2006) proposed a measure of general savings that reflected the extent to which any parallel processing is being carried out in a dual-task situation. The equation for general savings due to parallel processing is

$\mathrm{RT}_{\mathrm{T} 1-\mathrm{LONG}}+\mathrm{RT}_{\mathrm{T} 2-\mathrm{LONG}}-\left(\mathrm{SOA}+\mathrm{RT}_{\mathrm{T} 2-\mathrm{SHORT}}\right)$ 
where SOA is the shortest SOA, $\mathrm{RT}_{\mathrm{Tn}-\mathrm{LONG}}$ is the RT at the longest SOA for Task $\mathrm{n}$, and $\mathrm{RT}_{\mathrm{Tn} \text {-SHORT }}$ is the RT at the shortest SOA. The logic of Eq. 1 is that, if there were no parallel processing at all, we would expect the time from the onset of the Task 1 stimulus (S1) to the response to Task 2 (R2) to be

$\mathrm{S}_{1}$ to $\mathrm{R}_{2}=\mathrm{RT}_{\mathrm{T} 1-\mathrm{LONG}}+\mathrm{RT}_{\mathrm{T} 2-\mathrm{LONG}}$.

If there were perfectly parallel processing, then it would be

$\mathrm{S}_{1}$ to $\mathrm{R}_{2}=\mathrm{SOA}+\mathrm{RT}_{\mathrm{T} 2-\mathrm{LONG}}$.

The amount of parallel processing that is possible, then, would be

$$
\begin{gathered}
\mathrm{RT}_{\mathrm{T} 1-\mathrm{LONG}}+\mathrm{RT}_{\mathrm{T} 2-\mathrm{LONG}}-\left(\mathrm{SOA}+\mathrm{RT}_{\mathrm{T} 2-\mathrm{LONG}}\right) \\
=\mathrm{R}_{\mathrm{T} 1-\mathrm{LONG}}-\mathrm{SOA} .
\end{gathered}
$$

Finally, the proportion of the possible parallel processing that was observed with the shortest SOA of $50 \mathrm{~ms}$ would be

$\frac{R T_{T 1-L O N G}+R T_{T 2-L O N G}-R T_{T 2-S H O R T}-50}{R T_{T 1-L O N G}-50}$.

We calculated the measure in Eq. 5 for both Experiment 1 and Experiment 2. For Experiment 1, proportional parallel processing scores were calculated for both compatible and incompatible conditions. ANOVA was performed with age group as a between-subjects factor and compatibility as a within-subjects factor. Older participants who appeared to have been grouping were excluded from the analysis. The analysis showed a significant effect of age group, $F(1,39)=$ $20.39, p<0.001, \eta^{2}=0.33$, with proportional parallel processing much higher in older adults $(M=0.62, S E=0.07)$ than in younger adults $(M=0.18, S E=0.07)$. There also was a main effect of compatibility, $F(1,39)=9.35, p<0.001, \eta^{2}=0.18$, with greater parallel processing for compatible responses $(M=$ $0.46, S E=0.05)$ than for incompatible responses $(M=0.34$, $S E=0.06)$.

If slowed processing of Task 1 confers an advantage, then it should be seen not only in older adults but in slower younger adults as well. Consequently, because we had a relatively large number of younger adults in Experiment 2, we split them into faster and slower groups by standardizing the RTs to each of the two tasks at the longest SOA, summing those $\mathrm{z}$ scores, and splitting at the median sum. For Experiment 2, an ANOVA of parallel processing scores as a function of condition (incongruent, neutral, and congruent stimuli) and group (faster younger adults, slower younger adults, or older adults) found a significant main effect of group, $F(2,58)=3.49, p=0.04, \eta^{2}=0.11$, with older adults $(M=0.35, S E=0.10)$ displaying significantly more parallel processing than faster younger adults
$(M=-0.06, S E=0.10)$ and with slower younger adults $(M=0.19, S E=0.10)$ intermediate. There also was a significant main effect of type with congruent conditions $(M=0.28, S E=0.07)$ showing more parallel processing than incongruent $(M=0.04, S E=0.08)$, with neutral conditions $(M=0.16, S E=0.07)$ intermediate. Slowed processing does appear to allow for more parallel processing. In sum, it is at least possible that higher levels of parallel processing contributed to smaller-thanexpected PRP effects in the older group. Arguably, the prebottleneck, perceptual stage of Task 2 was being performed in parallel with Task 1.

\section{Conclusions}

Because there is so little evidence concerning backward compatibility effects in older adults, any conclusions must be cautious and preliminary. Age differences in response compatibility effects have been sometimes found (Allen et al., in press) and sometimes not (Experiment 1). When response compatibility effects have been found, they are of equivalent magnitude in younger and older adults. Age differences in stimulus compatibility effects have been sometimes found (Experiment 2) and sometimes not (Grabbe \& Allen, 2012). When stimulus compatibility effects have been found, they are of equivalent magnitude in younger and older adults. We might then argue that when compatibility involves direct response facilitation (in response compatibility) or highly practiced associations (in stimulus compatibility), effects will be seen in older adults. With so little evidence, that argument is ad hoc if not circular. We did find evidence in Experiment 2 consistent with the possibility that some aspects of central processing of Task 2 were performed in parallel with Task 1 . There was an effect of the color in Task 1 on the color word in Task 2 that was larger than could be accounted for by carryover from Task 1 to Task 2. If this reflects a bypass of the response-selection bottleneck, it is only partial. Some situations in which one or the other or both of the tasks are simple and very extensively practiced show indications in younger adults that the bottleneck can be completely bypassed (Maquestiaux et al., 2008; Ruthruff et al., 2006). Older adults failed to show these indications even after very extensive training (Maquestiaux, Laguë-Beauvais, Ruthruff, Hartley, \& Bherer, 2010; Maquestiaux, Didierjean, Ruthruff, Chauvel, \& Hartley, 2013). Older adults do show evidence of partial bypass when Task 2 stimuli and responses are ideomotor compatible (Hartley et al., 2015) or very strongly associated (Experiment 2, possibly), and the magnitude of the effect is the same as in younger adults (Hartley et al., 2015). This does converge with the ad hoc explanation that we offered for the discrepancies between our results and those of Grabbe and Allen (2012) and Allen et al. (in press). 
Acknowledgments The authors thank Jennifer Han, Alynn Hembrough, Shruti Kanan, Carolyn Lasch, and Gabriela Paganini for assistance with data collection.

Support for this research was provided by the Molly Mason Jones Fund

\section{Compliance with ethical standards}

Conflict of interest The authors declare that there are no conflicts of interest regarding the publication of this article.

\section{References}

Allen, P., Lien, M.-C., Murphy, M., Sanders, R., Judge, K., \& McCann, R. (2002). Age differences in overlapping-task performance: Evidence for efficient parallel processing in older adults. Psychology and Aging, 17, 505-519. doi:10.1037/0882-7974.17.3. 505

Allen, P. A., Lien, M.-C., \& Jardin, E. (in press). Age-related emotional bias in processing two emotionally valenced tasks. Psychological Research. doi: 10.1007/s00426-015-0711-8

Cleland, A., Gaskell, M., Quinlan, P., \& Tamminen, J. (2006). Frequency effects in spoken and visual word recognition: Evidence from dualtask methodologies. Journal of Experimental Psychology: Human Perception and Performance, 32, 104-119. doi:10.1037/00961523.32.1.104

Ellenbogen, R., \& Meiran, N. (2011). Objects and events as determinants of parallel processing in dual tasks: Evidence from the backward compatibility effect. Journal of Experimental Psychology: Human Perception and Performance, 37, 152-167. doi:10.1037/a0019958

Fagot, C., \& Pashler, H. (1992). Making two responses to a single object: Implications for the central attentional bottleneck. Journal of Experimental Psychology: Human Perception and Performance, 18, 1058-1079. doi:10.1037/0096-1523.18.4.1058

Gaskell, M., Quinlan, P., Tamminen, J., \& Cleland, A. (2008). The nature of phoneme representation in spoken word recognition. Journal of Experimental Psychology: General, 137, 282-302. doi:10.1037/ 0096-3445.137.2.282

Grabbe, J. W., \& Allen, P. A. (2012). Cross-task compatibility and agerelated dual-task performance. Experimental Aging Research, 38, 469-487. doi:10.1080/0361073X.2012.726154

Greenwald, A. G. (1972). On doing two things at once: Time sharing as a function of ideomotor compatibility. Journal of Experimental Psychology, 94, 52-57. doi:10.1037/h0032762

Greenwald, A. G. (2003). On doing two things at once: III. Confirmation of perfect timesharing when simultaneous tasks are ideomotor compatible. Journal of Experimental Psychology: Human Perception and Performance, 29, 859-868. doi:10.1037/0096-1523.29.5.859

Greenwald, A. G. (2004). On doing two things at once: IV. Necessary and sufficient conditions: Rejoinder to Lien, Proctor, and Ruthruff (2003). Journal of Experimental Psychology: Human Perception and Performance, 30, 632-636. doi:10.1037/0096-1523.30.3.632

Greenwald, A. G. (2005). A reminder about procedures needed to reliably produce perfect timesharing: Comment on Lien, McCann, Ruthruff, and Proctor (2005). Journal of Experimental Psychology: Human Perception and Performance, 31, 221-225. doi:10.1037/00961523.31.1.221

Greenwald, A. G., \& Shulman, H. G. (1973). On doing two things at once: II. Elimination of the psychological refractory period effect. Journal of Experimental Psychology, 101(1), 70-76. doi:10.1037/ h0035451
Hartley, A. A. (2001). Age differences in dual-task interference are localized to response generation processes. Psychology and Aging, 16, 47-54. doi:10.1037/0882-7974.16.1.47

Hartley, A. A., \& Little, D. M. (1999). Age-related differences and similarities in dual-task interference. Journal of Experimental Psychology: General, 128(4), 416-449. doi:10.1037/0096-3445. 128.4.416

Hartley, A. A., \& Maquestiaux, F. (2007). Success and failure at dual-task coordination by younger and older adults. Psychology and Aging, 22, 215-222. doi:10.1037/0882-7974.22.2.215

Hartley, A. A., Seaman, B., \& Maquestiaux, F. (2015). Ideomotorcompatible tasks partially escape dual-task interference in both young and elderly adults. Psychology and Aging, 30, 36-45.

Hommel, B. (1998). Automatic stimulus-response translation in dualtask performance. Journal of Experimental Psychology: Human Perception and Performance, 24, 1368-1384. doi:10.1037/00961523.24.5.1368

Hommel, B., \& Eglau, B. (2002). Control of stimulus-response translation in dual-task performance. Psychological Research, 66, 260 273. doi:10.1007/s00426-002-0100-y

Lien, M., Allen, P., Ruthruff, E., Grabbe, J., McCann, R. S., \& Remington, R. W. (2006). Visual word recognition without central attention: Evidence for greater automaticity with advancing age. Psychology and Aging, 21, 431-447. doi:10.1037/0882-7974.21.3. 431

Lien, M., McCann, R. S., Ruthruff, E., \& Proctor, R. W. (2005). Dual-task performance with ideomotor-compatible tasks: Is the central processing bottleneck intact, bypassed, or shifted in locus? Journal of Experimental Psychology: Human Perception and Performance, 31, 122-144. doi:10.1037/0096-1523.31.1.122

Lien, M., Proctor, R. W., \& Allen, P. A. (2002). Ideomotor compatibility in the psychological refractory period effect: 29 years of oversimplification. Journal of Experimental Psychology. Human Perception and Performance, 28, 396-409. doi:10.1037/0096-1523.28.2.396

Lien, M., Proctor, R. W., \& Ruthruff, E. (2003). Still no evidence for perfect timesharing with two ideomotor-compatible tasks: A reply to Greenwald (2003). Journal of Experimental Psychology: Human Perception and Performance, 29, 1267-1272. doi:10.1037/00961523.29.6.1267

MacLeod, C. M. (1991). Half a century of research on the Stroop effect: An integrative review. Psychological Bulletin, 109, 163-203. doi: 10.1037/0033-2909.109.2.163

Maquestiaux, F., Didierjean, A., Ruthruff, E., Chauvel, G., \& Hartley, A. A. (2013). Lost ability to automatize task performance in old age. Psychonomic Bulletin \& Review, 20, 1206-1212. doi:10.3758/ s13423-013-0438-8

Maquestiaux, F., Laguë-Beauvais, M., Ruthruff, E., Hartley, A., \& Bherer, L. (2010). Learning to bypass the central bottleneck: Declining automaticity with advancing age. Psychology and Aging, 25, 177-192. doi:10.1037/a0017122

Maquestiaux, F., Laguë-Beauvais, M., Ruthruff, E., \& Bherer, L. (2008). Bypassing the central bottleneck after single-task practice in the psychological refractory period paradigm: Evidence for task automatization and greedy resource recruitment. Memory \& Cognition, 36, 1262-1282. doi:10.3758/MC.36.7.1262

McCann, R. S., \& Johnston, J. C. (1992). Locus of the single-channel bottleneck in dual-task interference. Journal of Experimental Psychology: Human Perception and Performance, 18, 471-484. doi:10.1037/0096-1523.18.2.471

McCann, R. S., Remington, R. W., \& Van Selst, M. (2000). A dual-task investigation of automaticity in visual word processing. Journal of Experimental Psychology: Human Perception and Performance, 26, 1352-1370 doi:10.1037/0096-1523.26.4.1352

Miller, J. (2006). Backward crosstalk effects in psychological refractory period paradigms: Effects of second-task response types on first-task 
response latencies. Psychological Research, 70, 484-493. doi:10. 1007/s00426-005-0011-9

Miller, J., \& Alderton, M. (2006). Backward response-level crosstalk in the psychological refractory period paradigm. Journal of Experimental Psychology: Human Perception and Performance, 32, 149-165. doi:10.1037/0096-1523.32.1.14

Pashler, H. (1994). Dual-task interference in simple tasks: Data and theory. Psychological Bulletin, 116, 220-244. doi:10.1037/0033-2909. 116.2.220

Pashler, H. (1998). The psychology of attention. Cambridge, MA: MIT Press.

Pashler, H., Carrier, M., \& Hoffman, J. (1993). Saccadic eye movements and dual-task interference. Quarterly Journal of Experimental Psychology. A, Human Experimental Psychology, 46A, 51-82. doi: 10.1080/14640749308401067

Rabovsky, M., Álvarez, C., Hohlfeld, A., \& Sommer, W. (2008). Is lexical access autonomous? Evidence from combining overlapping tasks with recording event-related brain potentials. Brain Research, 1222, 156-165. doi:10.1016/j.brainres.2008.05.066

Ruthruff, E., Allen, P. A., Lien, M.-C., \& Grabbe, J. (2008). Visual word recognition without central attention: Evidence for greater automaticity with greater reading ability. Psychonomic Bulletin \& Review, 15, 337-343. doi:10.3758/PBR.15.2.337

Ruthruff, E., Van Selst, M., Johnston, J. C., \& Remington, R. (2006). How does practice reduce dual-task interference: Integration, automatization, or just stage-shortening? Psychological Research, 70, 125-142. doi:10.1007/s00426-004-0192-7

Schneider, W., Eschman, A., \& Zuccolotto, A. (2002). E-Prime user's guide. Pittsburgh: Psychology Software Tools Inc.

Spence, C. (2008). Cognitive neuroscience: Searching for the bottleneck in the brain. Current Biology, 18, R965-R968.
Stroop, J. (1935). Studies of interference in serial verbal reactions. Journal of Experimental Psychology, 18, 643-662. doi:10.1037/ h0054651

Telford, C. W. (1931). The refractory phase of voluntary and associative responses. Journal of Experimental Psychology, 14, 1-36. doi:10. 1037/h0073262

Thomson, S. J., \& Watter, S. (2013). Information continuity across the response selection bottleneck: Early parallel Task 2 response activation contributes to overt Task 2 performance. Attention, Perception, \& Psychophysics, 75, 934-953. doi:10.3758/s13414-013-0457-6

Thomson, S. J., Watter, S., \& Finkelshtein, A. (2010). Parallel response selection in dual-task situations via automatic category-to-response translation. Attention, Perception, \& Psychophysics, 72, 1791-1802. doi:10.3758/APP.72.7.1791

Tombu, M., \& Jolicœur, P. (2005). Testing the predictions of the central capacity sharing model. Journal of Experimental Psychology: Human Perception and Performance, 31, 790-802. doi:10.1037/ 0096-1523.31.4.790

Verhaeghen, P. (2014). Elements of cognitive aging. New York: Oxford University Press. doi:10.1093/acprof:oso/9780195368697.001. 0001

Vince, M. A. (1948). The intermittency of control movements and the psychological refractory period. British Journal of Psychology, 38, $149-157$.

Watter, S., \& Logan, G. D. (2006). Parallel response selection in dual-task situations. Perception \& Psychophysics, 68, 254-277. doi:10.3758/ BF03193674

Welford, A. T. (1952). The "psychological refractory period" and the timing of high-speed performance: A review and a theory. British Journal of Psychology, 43, 2-19.

Wheeler, D. D. (1970). Processes in word recognition. Cognitive Psychology, 1, 59-85. doi:10.1016/0010-0285(70)90005-8 